International Electronic Journal of Geometry

Volume 8 No. 2 PP. 9-20 (2015) C IEJG

\title{
ON THE SECOND KIND TWISTED SURFACES IN MINKOWSKI 3-SPACE
}

\author{
MILICA GRBOVIĆ, EMILIJA NEŠOVIĆ AND ANICA PANTIĆ \\ (Communicated by Kazım ILARSLAN)
}

\begin{abstract}
In this paper, we introduce the notion of the second kind twisted surfaces in Minkowski 3-space. We classify all non-degenerate second kind twisted surfaces in terms of flat, minimal, constant Gaussian and constant mean curvature surfaces, with respect to a chosen lightlike transversal bundle. We also prove that a lightlike second kind twisted surfaces, with respect to a chosen lightlike transversal vector bundle, are the lightcones, the lightlike binormal surfaces over pseudo null base curve and the lightlike ruled surfaces with null rulings whose base curve lies on lightcone.
\end{abstract}

\section{INTRODUCTION}

In the Euclidean 3-space, twisted surfaces are introduced by A. Gray in [5] to generalize the construction used to produce the Möbius strip and the twisted Klein bottle. These surfaces arise by rotating a profile curve lying in its supporting plane $\pi$ about a fixed point in $\pi$ (i.e. about an axis spanned by the orthogonal complement of $\pi$ ), while simultaneously the supporting plane $\pi$ rotates about an axis lying in it [2]. Hence twisted surfaces represent generalizations of the surfaces of the revolution and also can be called a double rotational surfaces. In the Euclidean and Minkowski space, twisted surfaces are classified in terms of flat, minimal, constant Gaussian and constant mean curvature surfaces in [2] and [3].

In Minkowski 3-space, the supporting plane $\pi$ of the twisted surface profile curve can be a spacelike, a timelike or a lightlike. If the supporting plane $\pi$ is a lightlike, the obtained twisted surfaces are only lightlike (lightlike planes or lightlike cones, see [4] page 5). This situation motivated us to introduce the second kind twisted surfaces in Minkowski 3-space, as a new kind of the twisted surfaces, whose profile curve lies in a lightlike supporting plane, but which can be a spacelike, a timelike and a lightlike. More precisely, we define the second kind twisted surfaces as the surfaces obtained by the rotation of a profile curve about an axis in its lightlike supporting plane $\pi$, while simultaneously the supporting plane $\pi$ rotates about an axis spanned by a lightlike transversal vector $\operatorname{lt}(T \pi)$ of $\pi$. We classify all non-degenerate second

Date: Received: May 17, 2015 and Accepted: August 12, 2015.

2010 Mathematics Subject Classification. 53C50, 53C40.

Key words and phrases. Twisted surface, Lightlike transversal bundle, Lorentzian rotation. 
kind twisted surfaces, with respect to a chosen lightlike transversal bundle, in terms of a flat, a minimal, constant Gaussian and constant mean curvature surfaces. We also prove that the lightlike second kind twisted surfaces, with respect to a chosen lightlike transversal vector bundle, are the lightcones, the lightlike binormal surfaces over pseudo null base curve and the lightlike ruled surfaces with null rulings, whose base curve lies on lightcone.

\section{Preliminaries}

Minkowski 3-space $E_{1}^{3}$ is a 3-dimensional affine space endowed with an indefinite flat metric $g$ with signature $(+,+,-)$. This means that metric bilinear form can be written as

$$
g(x, y)=x_{1} y_{1}+x_{2} y_{2}-x_{3} y_{3}
$$

for any two vectors $x=\left(x_{1}, x_{2}, x_{3}\right)$ and $y=\left(y_{1}, y_{2}, y_{3}\right)$ in $E_{1}^{3}$. Recall that a vector $v \in E_{1}^{3} \backslash\{0\}$ can be spacelike, timelike or null (lightlike), if $g(v, v)>0, g(v, v)<0$ and $g(v, v)=0$, respectively. A vector $v=0$ is said to be a spacelike. The norm of a vector $v$ is given by $\|v\|=\sqrt{|g(v, v)|}$. Two vectors $v$ and $w$ are said to be orthogonal, if $g(v, w)=0$. An arbitrary curve $\alpha$ in $E_{1}^{3}$, can locally be spacelike, timelike or null (lightlike), if all its velocity vectors $\alpha^{\prime}(s)$ are respectively spacelike, timelike or null ([7]). A spacelike curve $\alpha$ whose principal normal $N$ and binormal $B$ are null vectors satisfying $g(N, B)=1$, is called a pseudo null curve [9].

Lemma 2.1. Let $u, v$ and $w$ be the vectors in $E_{1}^{3}$. Then:

(i) $g(u \times v, w)=\operatorname{det}(u, v, w)$,

(ii) $u \times(v \times w)=-g(u, w) v+g(u, v) w$,

(iii) $g(u \times v, u \times v)=-g(u, u) g(v, v)+g(u, v)^{2}$.

Definition 2.1. A surface $S$ in $E_{1}^{3}$ is called a timelike (resp. a spacelike), if the induced metric on $S$ is a Lorentzian (resp. positive definite Riemmanian) metric.

Definition 2.2. A surface $S$ in $E_{1}^{3}$ is called a lightlike (degenerate or null), if the induced metric on $S$ is a degenerate.

The Gaussian curvature of a non-degenerate surface $x(s, t)$ in $E_{1}^{3}$ is given by

$$
K(s, t)=g(U, U) \frac{L N-M^{2}}{E G-F^{2}},
$$

where $E, F, G$ are the coefficients of the first fundamental form, $L, M, N$ are the coefficients of the second fundamental form and

$$
U(s, t)=\frac{x_{s} \times x_{t}}{\left\|x_{s} \times x_{t}\right\|}
$$

is the unit normal vector field on $x(s, t)$. A non-degenerate surface in $E_{1}^{3}$ is called flat, if its Gaussian curvature vanishes identically. A non-degenerate surface in $E_{1}^{3}$ is called minimal (or stationary), if its mean curvature

$$
H=\frac{E N-2 F M+G L}{2\left|E G-F^{2}\right|}
$$

vanishes identically. A timelike ruled surface with parametrization

$$
x(s, t)=\beta(s)+\operatorname{tn}(s)
$$

in Minkowski 3-space is called a null scroll, if $\beta$ is a null base curve and $n$ is a null vector field along $\beta$, such that $g\left(\beta^{\prime}(s), n(s)\right)=1([6])$. Moreover, any timelike ruled 
surface with null rulings in $E_{1}^{3}$, can be reparameterized as a null scroll ([6]). A null scroll having a non-zero constant Gaussian curvature $K$ and a non-zero constant mean curvature $H=K^{2}$, is called a $B$-scroll.

\section{The SECOND Kind TWISTED SURfaces IN $E_{1}^{3}$}

In this section, we introduce the notion of the second kind twisted surfaces in $E_{1}^{3}$. The profile curve of such surfaces lies in a lightlike supporting plane. In relation to that, denote by $\pi$ a lightlike plane in $E_{1}^{3}$. Since $\left.g\right|_{\pi}$ is degenerate on $\pi$, the radical (null) space of $T_{p} \pi$ at each point $P \in \pi$ is a subspace $\operatorname{Rad}\left(T_{p} \pi\right)$ defined by ([1])

$$
\operatorname{Rad}\left(T_{p} \pi\right)=\left\{Y \in T_{p} \pi \mid g(X, Y)=0, \forall X \in T_{p} \pi\right\} .
$$

For the radical space it holds

$$
\operatorname{Rad}\left(T_{p} \pi\right)=T_{p} \pi \cap T_{p} \pi^{\perp}
$$

Denote by $S(T \pi)$ a complementary vector bundle of $\operatorname{Rad}(T \pi)$ in $\pi$. This means that

$$
T \pi=\operatorname{Rad}(T \pi) \oplus S(T \pi) .
$$

A vector bundle $S(T \pi)$ is called a screen distribution on $\pi$. For a given screen distribution $S(T \pi)$, it exists a unique complementary vector bundle $l \operatorname{tr}(T \pi)$ to $T \pi$ in $\left.T E_{1}^{3}\right|_{\pi}$. The vector bundle $\operatorname{tr}(T \pi)$ is called a lightlike transversal vector bundle of $\pi$. Consequently, we have the following decomposition ([1])

$$
\left.E_{1}^{3}\right|_{\pi}=\operatorname{Rad}(T \pi) \oplus S(T \pi) \oplus \operatorname{ltr}(T \pi) .
$$

Definition 3.1. The second kind twisted surface in Minkowski 3-space is a surface obtained by rotating the profile curve about an axis in its lightlike supporting plane $\pi$, while simultaneously the supporting plane $\pi$ rotates about an axis spanned by lightlike transversal vector $\operatorname{lt}(T \pi)$ of $\pi$.

According to Definition 3.1, the second kind twisted surfaces are a new generalization of the surfaces of revolution in Minkowski 3-space. It is known that the lightlike transversal vector bundle $\operatorname{lt}(T \pi)$ of a lightlike supporting plane $\pi$ is not unique ([1]). For example, if a lightlike supporting plane $\pi$ has the equation $x_{1}=x_{3}$, then $\operatorname{Rad}(T \pi)=\operatorname{span}\{(1,0,1)\}$, so the lightlike transversal bundle of $\pi$ can be spanned by $b=\left(\frac{1}{2}, 0,-\frac{1}{2}\right)$, or by $c=(0,1,-1)$, which shows that it is not unique. This implies that different choices of a lightlike transversal vector bundle of the supporting plane produce different parameterizations of the second kind twisted surfaces.

Denote by $\left\{e_{1}, e_{2}, e_{3}\right\}$ positively oriented pseudo-orthonormal basis of $E_{1}^{3}$, consisting of the unit vector $e_{1}=(0,1,0)$ and of two normalized null vectors $e_{2}=$ $(1,0,1)$ and $e_{3}=\left(\frac{1}{2}, 0,-\frac{1}{2}\right)$. Up to isometries of $E_{1}^{3}$, assume that a lightlike supporting plane $\pi$ of the profile curve has the equation $x_{1}=x_{3}$. Then the radical space $\operatorname{Rad}(T \pi)$ of $\pi$ is spanned by $e_{2}=(1,0,1)$. Let us choose a lightlike transversal bundle $\operatorname{lt}(T \pi)$ of $\pi$ as follows. Consider a locally defined non-zero section $a=(1,0,0)$, defined on an open set $U \subset E_{1}^{3}$. Then $g\left(a, e_{2}\right)=1, g(a, a)=1$, so the lightlike transversal bundle $\operatorname{ltr}(T \pi)$ is spanned by ([1]):

$$
b=\frac{1}{g\left(a, e_{2}\right)}\left[a-\frac{g(a, a)}{2 g\left(a, e_{2}\right)} e_{2}\right]=\left(\frac{1}{2}, 0,-\frac{1}{2}\right)=e_{3} .
$$

A chosen lightlike transversal vector $e_{3}$ will span one of two axis of two simultaneous rotations producing the second kind twisted surface. 
Since the profile curve $\alpha$ of the second kind twisted surface lies in $\pi$, it can be parameterized as

$$
\alpha(t)=(t, g(t), t),
$$

for some differentiable function $g(t)$. In a lightlike plane there are no timelike straight lines, so the profile curve $\alpha$ can rotate about a spacelike or a lightlike axis lying in $\pi$. Consequently, we may distinguish two cases: (3.A) an axis $l$ is a spacelike; (3.B) an axis $l$ is a lightlike.

(3.A) an axis $l$ is a spacelike;

By rotating the profile curve $\alpha$ about an axis $l$ through the point $P(a, 0, a)$, parallel with the spacelike vector $e_{1}=(0,1,0)$, we obtain parametrization

$$
\bar{x}(s, t)=\left[\begin{array}{l}
a \\
0 \\
a
\end{array}\right]+\left[\begin{array}{ccc}
\cosh (b s) & 0 & \sinh (b s) \\
0 & 1 & 0 \\
\sinh (b s) & 0 & \cosh (b s)
\end{array}\right]\left[\begin{array}{c}
t \\
g(t) \\
t
\end{array}\right],
$$

where $\phi=b s, b \in R$ is the Lorentzian angle of rotation. By using (3.2) and applying the simultaneous rotation of the supporting plane $\pi$ about a lightlike axis spanned by the lightlike transversal vector $e_{3}$, we find that parametrization of the second kind twisted surface reads

$$
x(s, t)=\left[\begin{array}{ccc}
1-\frac{s^{2}}{2} & s & -\frac{s^{2}}{2} \\
-s & 1 & -s \\
\frac{s^{2}}{2} & -s & 1+\frac{s^{2}}{2}
\end{array}\right]\left[\begin{array}{c}
a+t \cosh (b s)+t \sinh (b s) \\
g(t) \\
a+t \cosh (b s)+t \sinh (b s)
\end{array}\right] .
$$

Since both rotations are executed simultaneously, we use the same parameter $s$ in relation (3.3). If $b=0$, the first rotation is identity mapping. Consequently, assume that $b \in R \backslash\{0\}$.

By using (3.3), a straightforward calculation shows that the coefficients $E, F, G$ of the first fundamental form of $x(s, t)$ are given by

$$
\begin{aligned}
& E(s, t)=g\left(x_{s}, x_{s}\right)=4\left(a+t e^{b s}\right)^{2}+4 b t g(t) e^{b s}, \\
& F(s, t)=g\left(x_{s}, x_{t}\right)=2 g(t) e^{b s}-2 g^{\prime}(t)\left(a+t e^{b s}\right), \\
& G(s, t)=g\left(x_{t}, x_{t}\right)=g^{\prime 2}(t) .
\end{aligned}
$$

If $E G-F^{2}=0$, by using (3.4) we obtain the system of equations

$$
2 t g(t) g^{\prime}(t)-g(t)^{2}=0, \quad b t g(t) g^{\prime 2}(t)+2 a g(t) g^{\prime}(t)=0 .
$$

The only solutions of the above system are: (1) $g(t)=a=0$; (2) $g(t)=0, a \neq 0$.

(1) If $g(t)=a=0$, substituting this in (3.3) we obtain that the second kind twisted surface is a lightcone with parametrization

$$
x(s, t)=t\left(\left(1-s^{2}\right) e^{b s},-2 s e^{b s},\left(1+s^{2}\right) e^{b s}\right) .
$$

(2) If $g(t)=0$ and $a \neq 0$, substituting this in (3.3) we get that the second kind twisted surface is a ruled surface with parametrization

$$
x(s, t)=\left(\left(1-s^{2}\right) a,-2 a s,\left(1+s^{2}\right) a\right)+t\left(\left(1-s^{2}\right) e^{b s},-2 s e^{b s},\left(1+s^{2}\right) e^{b s}\right),
$$

where $\alpha(s)=\left(\left(1-s^{2}\right) a,-2 a s,\left(1+s^{2}\right) a\right)$ is a pseudo null base curve. It can be easily verified that a binormal vector of $\alpha$ is a null vector given by

$$
B(s)=\operatorname{sgn}(a)\left(\frac{s^{2}-1}{2}, s,-\frac{s^{2}+1}{2}\right) .
$$


Consequently, the rulings

$$
\beta(s)=e^{b s}\left(1-s^{2},-2 s, 1+s^{2}\right)
$$

of the ruled surface with parametrization (3.5) have the same direction as the binormal vector $B(s)$ of $\alpha$. Putting $\beta(s)=B(s)$ in parametrization (3.5), we obtain parametrization

$$
x(s, t)=\left(\left(1-s^{2}\right) a,-2 a s,\left(1+s^{2}\right) a\right)+t\left(\frac{s^{2}-1}{2}, s,-\frac{s^{2}+1}{2}\right) .
$$

of the binormal surface over a pseudo null base curve. This proves the next theorem.

Theorem 3.1. A lightlike second kind twisted surfaces in $E_{1}^{3}$ with parametrization (3.3) are the lightcones

$$
x(s, t)=t\left(\left(1-s^{2}\right) e^{b s},-2 s e^{b s},\left(1+s^{2}\right) e^{b s}\right), \quad b \in R_{0},
$$

and the lightlike binormal surfaces

$$
x(s, t)=\left(\left(1-s^{2}\right) a,-2 a s,\left(1+s^{2}\right) a\right)+t\left(\frac{s^{2}-1}{2}, s,-\frac{s^{2}+1}{2}\right), \quad a \in R_{0},
$$

over a pseudo null base curve (figure 1).

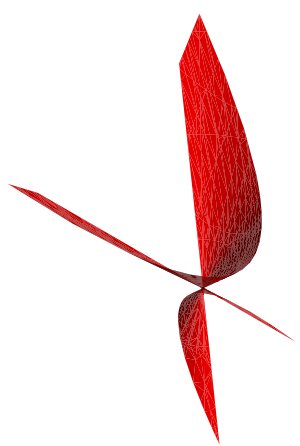

Figure 1. A lightlike binormal surface over a pseudo null base curve

In order to classify a non-degenerate second kind twisted surfaces with parametrization (3.3) in terms of flat and minimal surfaces, we obtain that the coefficients

$$
L(s, t)=\frac{\left[x_{s s}, x_{s}, x_{t}\right]}{\left\|x_{s} \times x_{t}\right\|}, \quad M(s, t)=\frac{\left[x_{s t}, x_{s}, x_{t}\right]}{\left\|x_{s} \times x_{t}\right\|}, \quad N(s, t)=\frac{\left[x_{t t}, x_{s}, x_{t}\right]}{\left\|x_{s} \times x_{t}\right\|},
$$

of the second fundamental form are given by

$$
\begin{aligned}
L(s, t)= & \frac{1}{W(s, t)}\left[8 t^{2} e^{3 b s}+e^{2 b s}\left(8 b t g(t)+16 a t+4 b t^{2} g^{\prime}(t)\right)\right. \\
& \left.+e^{b s}\left(8 a^{2}+4 a b t g^{\prime}(t)+2 b^{2} t g(t) g^{\prime}(t)\right)\right], \\
M(s, t)= & \frac{1}{W(s, t)}\left[e^{2 b s}\left(4 g(t)-4 t g^{\prime}(t)\right)+e^{b s}\left(2 b g(t) g^{\prime}(t)-4 a g^{\prime}(t)\right.\right. \\
& \left.\left.-2 b t g^{\prime 2}(t)\right)\right] \\
N(s, t)= & \frac{1}{W(s, t)}(-2) g(t) g^{\prime \prime}(t) e^{b s}
\end{aligned}
$$


where $W(s, t)$ is given by

$$
W(s, t)=\left\|x_{s} \times x_{t}\right\|=\sqrt{\left|E G-F^{2}\right|} .
$$

Theorem 3.2. The second kind twisted surface in $E_{1}^{3}$ with parametrization (3.3) is flat, if and only if it is a part of a cone

$$
x(s, t)=t\left(\left(1-s^{2}\right) e^{b s}+c s, c-2 s e^{b s},\left(1+s^{2}\right) e^{b s}-c s\right),
$$

whose base curve lies on a pseudosphere $S_{1}^{2}(c), c \in R_{0}^{+}, b \in R_{0}$.

Proof. By using the relation (3.6), it follows that $L N-M^{2}=0$ if and only if the next system of equations is satisfied:

$$
\begin{aligned}
-16 g(t) g^{\prime \prime}(t) t^{2}-\left(4 g(t)-4 t g^{\prime}(t)\right)^{2} & =0, \\
-2 g(t) g^{\prime \prime}(t)\left(8 b t g(t)+16 a t+4 b t^{2} g^{\prime}(t)\right)-2(4 g(t) & - \\
\left.4 t g^{\prime}(t)\right)\left(2 b g(t) g^{\prime}(t)-4 a g^{\prime}(t)-2 b t g^{\prime 2}(t)\right) & =0, \\
-2 g(t) g^{\prime \prime}(t)\left(8 a^{2}+4 a b t g^{\prime}(t)+2 b^{2} t g(t) g^{\prime}(t)\right) & - \\
\left(2 b g(t) g^{\prime}(t)-4 a g^{\prime}(t)-2 b t g^{\prime 2}(t)\right)^{2} & =0 .
\end{aligned}
$$

The only solution of the above system of equations is $g(t)=c t, c \neq 0$ and $a=0$. Substituting this in (3.3), we obtain parametrization (3.7). Consequently, the second kind twisted surface is a cone with vertex at the origin, over the pseudospherical curve

$$
\alpha(s)=\left(\left(1-s^{2}\right) e^{b s}+c s, c-2 s e^{b s},\left(1+s^{2}\right) e^{b s}-c s\right) .
$$

Theorem 3.3. There are no minimal second kind twisted surfaces in $E_{1}^{3}$ with parametrization (3.3).

Proof. Assume that it exists minimal second kind twisted surface in $E_{1}^{3}$, with parametrization (3.3). By using the relations (3.4) and (3.6), it follows that $E N-$ $2 F M+G L=0$ if and only if the next system of equations is satisfied:

$$
\begin{aligned}
-8 g(t) g^{\prime \prime}(t) t^{2}+8 t^{2} g^{\prime 2}(t)-\left(4 g(t)-4 t g^{\prime}(t)\right)^{2} & =0, \\
-2 g(t) g^{\prime \prime}(t)(8 a t+4 b t g(t))+8 b t g(t) g^{\prime 2}(t)+16 a t g^{\prime 2}(t) & + \\
4 b t^{2} g^{\prime 3}(t)-\left(4 g(t)-4 t g^{\prime}(t)\right)\left(2 b g(t) g^{\prime}(t)-8 a g^{\prime}(t)\right. & - \\
\left.2 b t g^{\prime 2}(t)\right) & =0 . \\
-8 g(t) g^{\prime \prime}(t) a^{2}+g^{\prime 2}(t)\left(8 a^{2}+4 a b t g^{\prime}(t)+2 b^{2} t g(t) g^{\prime}(t)\right) & + \\
4 a g^{\prime}(t)\left(2 b g(t) g^{\prime}(t)-4 a g^{\prime}(t)-2 b t g^{\prime 2}(t)\right) & =0 .
\end{aligned}
$$

The only solution of the above system of equations is $g(t)=0$. Then relation (3.4) implies $E G-F^{2}=0$, which is a contradiction.

Theorem 3.4. The second kind twisted surface in $E_{1}^{3}$ with parametrization (3.3) has a non-zero constant Gaussian curvature $K=c$, if and only if it is a part of: (a) a B-scroll with parametrization

$$
x(s, t)=\left(\frac{s}{\sqrt{c}}+\frac{\sqrt{c}}{2} t\left(1-s^{2}\right), \frac{1}{\sqrt{c}}-\sqrt{c} s t,-\frac{s}{\sqrt{c}}+\frac{\sqrt{c}}{2} t\left(1+s^{2}\right)\right),
$$

where $c \in R_{0}^{+}$; 
(b) a pseudosphere $S_{1}^{2}\left(\frac{1}{\sqrt{c}}\right)$ with parametrization

$$
\begin{aligned}
x(s, t)= & \left(\left(1-s^{2}\right)\left(a+t e^{b s}\right)+\frac{s}{\sqrt{c}},-2 s\left(a+t e^{b s}\right)+\frac{1}{\sqrt{c}},\right. \\
& \left.\left(1+s^{2}\right)\left(a+t e^{b s}\right)-\frac{s}{\sqrt{c}}\right),
\end{aligned}
$$

where $c \in R_{0}^{+}$and $a \in R_{0}$.

Proof. Assume that it exists the second kind twisted surface in $E_{1}^{3}$ with parametrization (3.3) and a non-zero constant Gaussian curvature $K=c \in R_{0}$. According to (2.1), it holds

$$
L N-M^{2}=c g(U, U)\left(E G-F^{2}\right) .
$$

Since $\operatorname{sgn} g(U, U)=-\operatorname{sgn}\left(E G-F^{2}\right)$ and substituting (3.4) and (3.6) in (3.16), we obtain the system of equations:

$$
\begin{aligned}
& 16 g(t) g^{\prime \prime}(t) t^{2}+\left[4 g(t)-4 t g^{\prime}(t)\right]^{2}=c\left[8 g(t) g^{\prime}(t) t-4 g^{2}(t)\right]^{2}, \\
& -2 g(t) g^{\prime \prime}(t)\left[8 b t g(t)+16 a t+4 b t^{2} g^{\prime}(t)\right]-2\left[4 g(t)-4 t g^{\prime}(t)\right] \\
& {\left[2 b g(t) g^{\prime}(t)-4 a g^{\prime}(t)-2 b t g^{2}(t)\right]=-2 c\left[8 g(t) g^{\prime}(t) t-\right.} \\
& \left.4 g^{2}(t)\right]\left[4 b t g(t) g^{\prime 2}(t)+8 a g(t) g^{\prime}(t)\right], \\
& -2 g(t) g^{\prime \prime}(t)\left[8 a^{2}+4 a b t g^{\prime}(t)+2 b^{2} t g(t) g^{\prime}(t)\right]-\left[2 b g(t) g^{\prime}(t)-\right. \\
& \left.4 a g^{\prime}(t)-2 b t g^{\prime 2}(t)\right]^{2}=-c\left[4 b t g(t) g^{\prime 2}(t)+8 a g(t) g^{\prime}(t)\right]^{2} .
\end{aligned}
$$

The only solutions of the above system of equations are (a) $g(t)=\frac{1}{\sqrt{c}}, c \in R_{0}^{+}$, $a=0 ;(\mathrm{b}) g(t)=\frac{1}{\sqrt{c}}, c \in R_{0}^{+}, a \neq 0$;

(a) If $g(t)=\frac{1}{\sqrt{c}}, c \in R_{0}^{+}$and $a=0$, substituting this in relation (3.3), we obtain parametrization of the ruled surface

$$
x(s, t)=\left(\left(1-s^{2}\right) t e^{b s}+\frac{s}{\sqrt{c}},-2 s t e^{b s}+\frac{1}{\sqrt{c}},\left(1+s^{2}\right) t e^{b s}-\frac{s}{\sqrt{c}}\right)
$$

with null base curve

$$
\alpha(s)=\left(\frac{s}{\sqrt{c}}, \frac{1}{\sqrt{c}},-\frac{s}{\sqrt{c}}\right)
$$

and null rulings

$$
\beta(s)=e^{b s}\left(1-s^{2},-2 s, 1+s^{2}\right) .
$$

Normalizing the rulings, we may assume $g\left(\alpha^{\prime}(s), \beta(s)\right)=1$. Hence the ruled surface is a $B$-scroll with parametrization (3.14) (figure 2). This proves statement (a).

(b) If $a \neq 0$ and $g(t)=\frac{1}{\sqrt{c}}$, substituting this in relation (3.3) we get parametrization (3.15). In this case, the second kind twisted surface is a pseudosphere $S_{1}^{2}\left(\frac{1}{\sqrt{c}}\right)$ with center at the origin, which proves (b). 


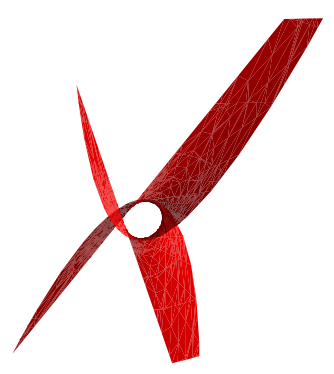

Figure 2. B-scroll

The following theorem can be proved analogously, so we omit its proof.

Theorem 3.5. The second kind twisted surface in $E_{1}^{3}$ with parametrization (3.3) has a non-zero constant mean curvature $H=h$, if and only if it is a part of:

(a) a B-scroll with parametrization

$$
x(s, t)=\left(\frac{s}{h}+\frac{h}{2} t\left(1-s^{2}\right), \frac{1}{h}-h s t,-\frac{s}{h}+\frac{h}{2} t\left(1+s^{2}\right)\right),
$$

where $h \in R_{0}^{+}$;

(b) a pseudosphere $S_{1}^{2}(h)$ with parametrization

$$
x(s, t)=\left(\left(1-s^{2}\right)\left(a+t e^{b s}\right)+\frac{s}{h},-2 s\left(a+t e^{b s}\right)+\frac{1}{h},\left(1+s^{2}\right)\left(a+t e^{b s}\right)-\frac{s}{h}\right),
$$

where $h \in R_{0}^{+}$and $a \in R_{0}$.

(3.B) an axis $l$ is a lightlike;

By rotating the profile curve $\alpha$ about an axis $l$ through the point $P(0, a, 0)$ parallel with a null vector $e_{2}=(1,0,1)$, we obtain parametrization

$$
\bar{x}(s, t)=\left[\begin{array}{l}
0 \\
a \\
0
\end{array}\right]+\left[\begin{array}{ccc}
1-\frac{b^{2} s^{2}}{2} & b s & \frac{b^{2} s^{2}}{2} \\
-b s & 1 & b s \\
-\frac{b^{2} s^{2}}{2} & b s & 1+\frac{b^{2} s^{2}}{2}
\end{array}\right]\left[\begin{array}{c}
t \\
g(t) \\
t
\end{array}\right] .
$$

If $b=0$, the rotation matrix in relation (3.20) is identity matrix. Hence assume $b \in$ $R \backslash\{0\}$. By using (3.20) and applying the simultaneous rotation of the supporting plane $\pi$ about an axis spanned by a null transversal vector $e_{3}=\left(\frac{1}{2}, 0,-\frac{1}{2}\right)$, we get that parametrization of the second kind twisted surface reads

$$
x(s, t)=\left[\begin{array}{ccc}
1-\frac{s^{2}}{2} & s & -\frac{s^{2}}{2} \\
-s & 1 & -s \\
\frac{s^{2}}{2} & -s & 1+\frac{s^{2}}{2}
\end{array}\right]\left[\begin{array}{c}
t+b s g(t) \\
a+g(t) \\
t+b s g(t)
\end{array}\right] .
$$

Since both rotations are realized simultaneously, we use the same parameter $s$ in (3.21). We also use the factor $b \neq 0$ in rotation matrix to indicate the fact that the speeds of the rotations can be different. 
By using (3.21), a straightforward calculation shows that the coefficients $E, F, G$ of the first fundamental form of $x(s, t)$ are given by

$$
\begin{aligned}
& E(s, t)=g\left(x_{s}, x_{s}\right)=4 b^{2} s^{2} g(t)^{2}+8 b s t g(t)+4 t^{2}+4 a b g(t)+4 b g(t)^{2}, \\
& F(s, t)=g\left(x_{s}, x_{t}\right)=2 a b s g^{\prime}(t)+2 a+2 g(t)-2 t g^{\prime}(t), \\
& G(s, t)=g\left(x_{t}, x_{t}\right)=g^{\prime 2}(t) .
\end{aligned}
$$

If $E G-F^{2}=0$, by using (3.22) we obtain system of equations:

$$
\begin{aligned}
g(t)^{2} g^{2}(t)-a^{2} g^{2}(t) & =0, \\
8 b t g(t) g^{2}(t)-4 a b g^{\prime}(t)\left(2 a+2 g(t)-2 t g^{\prime}(t)\right) & =0, \\
g^{2}(t)\left(4 t^{2}+4 a b g(t)+4 b g(t)^{2}\right)-\left(2 a+2 g(t)-2 t g^{\prime}(t)\right)^{2} & =0 .
\end{aligned}
$$

The only solutions of the above system of equations are (1) $g(t)=a=0 ;(2)$ $g(t)=-a, a \neq 0$.

(1) If $g(t)=a=0$, the axis $l$ coincide with the profile curve $\alpha$, which is a contradiction.

(2) If $g(t)=-a, a \neq 0$, substituting this in (3.21) we obtain that the second kind twisted surface is a lightlike ruled surface with parametrization

$$
x(s, t)=a b\left(s\left(s^{2}-1\right), 2 s^{2}, s\left(-1-s^{2}\right)\right)+t\left(1-s^{2},-2 s, 1+s^{2}\right),
$$

where $\alpha(s)=a b\left(s\left(s^{2}-1\right), 2 s^{2}, s\left(-1-s^{2}\right)\right)$ is a spacelike base curve lying on a lightcone. It can be easily verified that the rulings of the surface are the null vectors. This proves the following theorem.

Theorem 3.6. A lightlike second kind twisted surfaces in $E_{1}^{3}$ with parametrization (3.21) are the lightlike ruled surfaces

$$
x(s, t)=a b\left(s\left(s^{2}-1\right), 2 s^{2}, s\left(-1-s^{2}\right)\right)+t\left(1-s^{2},-2 s, 1+s^{2}\right), \quad a, b \in R_{0},
$$

with null rulings, whose base curve lies on a lightcone (figure 3).

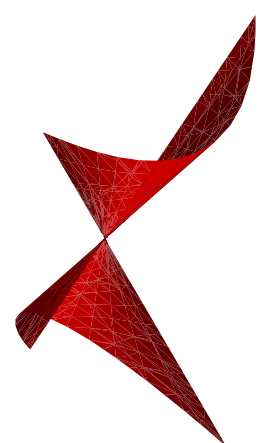

Figure 3. A lightlike ruled surface with null rulings

In order to classify a non-degenerate second kind twisted surfaces with parametrization (3.21) in terms of flat and minimal surfaces, we obtain that the coefficients 
$L, M$ and $N$ of the second fundamental form are given by

$$
\begin{aligned}
L(s, t) & =\frac{1}{W(s, t)}\left[8 b^{3} s^{3} g^{2}(t) g^{\prime}(t)+s^{2}\left(16 b^{2} t g(t) g^{\prime}(t)+8 b^{2} g^{2}(t)\right)\right. \\
& +s\left(16 b t g(t)+8 b t^{2} g^{\prime}(t)+12 b^{2} g^{2}(t) g^{\prime}(t)+8 a b^{2} g(t) g^{\prime}(t)\right) \\
& \left.+8 t^{2}+4 b t g(t) g^{\prime}(t)+8 a b g(t)+8 b g^{2}(t)\right], \\
M(s, t) & =\frac{1}{W(s, t)}\left[4 a b^{2} g^{\prime 2}(t) s^{2}+4 b s\left(g(t) g^{\prime}(t)-t g^{2}(t)+2 a g^{\prime}(t)\right)\right. \\
& \left.-4 t g^{\prime}(t)+2 a b g^{2}(t)+4 g(t)+4 a\right], \\
N(s, t) & =\frac{1}{W(s, t)}(-2) g^{\prime \prime}(t)(a+g(t)),
\end{aligned}
$$

where $W(s, t)=\left\|x_{s} \times x_{t}\right\|=\sqrt{\left|E G-F^{2}\right|}$.

Theorem 3.7. There are no flat the second kind twisted surfaces in $E_{1}^{3}$ with parametrization (3.21).

Proof. Assume that it exists flat the second kind twisted surface in $E_{1}^{3}$, with parametrization (3.21). By using the relation (3.23), it follows that $L N-M^{2}=0$ if and only if the following system of equations is satisfied:

$$
\begin{aligned}
& 16 a^{2} b^{4} g^{\prime 4}(t)=0 \\
& -16 b^{3} g^{2}(t) g^{\prime}(t) g^{\prime \prime}(t)(a+g(t))-32 a b^{3} g^{2}(t)\left(g(t) g^{\prime}(t)-\right. \\
& \left.t g^{\prime 2}(t)+2 a g^{\prime}(t)\right)=0 \\
& -2 g^{\prime \prime}(t)(a+g(t))\left(16 b^{2} t g(t) g^{\prime}(t)+8 b^{2} g^{2}(t)\right)-16\left(b g(t) g^{\prime}(t)-\right. \\
& \left.b t g^{\prime 2}(t)+2 a b g^{\prime}(t)\right)^{2}-8 a b^{2} g^{\prime 2}(t)\left(-4 t g^{\prime}(t)+\right. \\
& \left.2 a b g^{\prime 2}(t)+4 g(t)+4 a\right)=0, \\
& -2 g^{\prime \prime}(t)(a+g(t))\left(16 b t g(t)+8 b t^{2} g^{\prime}(t)+12 b^{2} g^{2}(t) g^{\prime}(t)+\right. \\
& \left.8 a b^{2} g(t) g^{\prime}(t)\right)-8\left(b g(t) g^{\prime}(t)-t b g^{2}(t)+2 a b g^{\prime}(t)\right)\left(-4 t g^{\prime}(t)+\right. \\
& \left.2 a b g^{\prime 2}(t)+4 g(t)+4 a\right)=0, \\
& -2 g^{\prime \prime}(t)(a+g(t))\left(8 t^{2}+4 b t g(t) g^{\prime}(t)+8 a b g(t)+8 b g^{2}(t)\right)- \\
& \left(-4 t g^{\prime}(t)+2 a b g^{\prime 2}(t)+4 g(t)+4 a\right)^{2}=0 .
\end{aligned}
$$

The only solution of the above system of equations is $g(t)=-a$, which implies that the surface is a lightlike. A contradiction.

Theorem 3.8. There are no minimal the second kind twisted surfaces in $E_{1}^{3}$ with parametrization (3.21).

Proof. Assume that it exists minimal the second kind twisted surface in $E_{1}^{3}$, with parametrization (3.21). By using the relations (3.22) and (3.23), it follows that $E N-2 F M+G L=0$ if and only if the next system of equations is satisfied:

$$
\begin{aligned}
& b^{3} g^{2}(t) g^{\prime 3}(t)-2 a^{2} b^{3} g^{\prime 3}(t)=0, \\
& g^{\prime \prime}(t) b^{2}(a+g(t)) g^{2}(t)-2 b^{2} t g(t) g^{\prime 3}(t)-b^{2} g^{2}(t) g^{2}(t)+ \\
& 2 a b^{2} g^{\prime}(t)\left[g(t) g^{\prime}(t)-t g^{\prime 2}(t)+2 a g^{\prime}(t)\right]+a b^{2} g^{\prime 2}(t)(2 a+ \\
& \left.2 g(t)-2 t g^{\prime}(t)\right)=0,
\end{aligned}
$$




$$
\begin{aligned}
& -4 b g^{\prime \prime}(t)(a+g(t)) t g(t)+4 b t g(t) g^{2}(t)+2 b t^{2} g^{3}(t)+ \\
& 3 b^{2} g(t)^{2} g^{\prime 3}(t)+2 a b^{2} g(t) g^{\prime 3}(t)-a b g^{\prime}(t)\left(-4 t g^{\prime}(t)+\right. \\
& \left.2 a b g^{\prime 2}(t)+4 g(t)+4 a\right)-2 b\left(2 a+2 g(t)-2 t g^{\prime}(t)\right)\left(g(t) g^{\prime}(t)-\right. \\
& \left.t g^{\prime 2}(t)+2 a b g^{\prime}(t)\right)=0, \\
& -2 g^{\prime \prime}(t)(a+g(t))\left(4 t^{2}+4 a b g(t)+4 b g(t)^{2}\right)+g^{2}(t)\left(8 t^{2}+\right. \\
& \left.4 b t g(t) g^{\prime}(t)+8 a b g(t)+8 b g^{2}(t)\right)-4\left(a+g(t)-t g^{\prime}(t)\right)\left(-4 t g^{\prime}(t)+\right. \\
& \left.2 a b g^{\prime 2}(t)+4 g(t)+4 a\right)=0 .
\end{aligned}
$$

The only solution of the above system of equations is $g(t)=-a$. This implies that the surface is a lightlike, which is a contradiction.

Theorem 3.9. The second kind twisted surface in $E_{1}^{3}$ with parametrization (3.21) has a non-zero constant Gaussian curvature $K=c$, if and only if it is a part of pseudosphere $S_{1}^{2}\left(\frac{\sqrt{c}}{c}\right)$ with parametrization

$$
\begin{aligned}
& x(s, t)=\left(\left(1-s^{2}\right)\left(t+b s\left(\frac{\sqrt{c}}{c}-a\right)\right)+s \frac{\sqrt{c}}{c}, \frac{\sqrt{c}}{c}-2 s\left(t+b s\left(\frac{\sqrt{c}}{c}-a\right)\right),\right. \\
& \left.\left(1+s^{2}\right)\left(t+b s\left(\frac{\sqrt{c}}{c}-a\right)\right)-s \frac{\sqrt{c}}{c}\right),
\end{aligned}
$$

where $a \in R, b \in R_{0}, c \in R_{0}^{+}$and $\frac{\sqrt{c}}{c} \neq a$.

Proof. Assume that it exists the second kind twisted surface in $E_{1}^{3}$ with parametrization (3.21) and a non-zero constant Gaussian curvature $K=c \in R_{0}$. According to $(2.1)$, it holds

$$
L N-M^{2}=c g(U, U)\left(E G-F^{2}\right) .
$$

Since $\operatorname{sgn} g(U, U)=-\operatorname{sgn}\left(E G-F^{2}\right)$ and substituting (3.22) and (3.23) in (3.34), we obtain the system of equations:

$$
\begin{gathered}
16 a^{2} b^{4} g^{\prime 4}(t)=c\left(4 b^{2} g^{2} g^{\prime 2}-4 a^{2} b^{2} g^{\prime 2}\right)^{2} \\
16 b^{3} g(t)^{2} g^{\prime}(t) g^{\prime \prime}(t)(a+g(t))+32 a b^{3} g^{2}(t)\left(g(t) g^{\prime}(t)-\right. \\
\left.t g^{\prime 2}(t)+2 a g^{\prime}(t)\right)=c\left(8 b^{2} g^{2}(t) g^{\prime 2}(t)-8 a^{2} b^{2} g^{\prime 2}(t)\right) \\
\left(8 b t g(t) g^{\prime 2}(t)-4 a b g^{\prime}(t)\left(2 a+2 g(t)-2 t g^{\prime}(t)\right)\right) \\
2 g^{\prime \prime}(a+g(t))\left(16 b t g(t) g^{\prime}(t)+8 b^{2} g(t)^{2}\right)+16\left(b g(t) g^{\prime}(t)-\right. \\
\left.b t g^{\prime 2}(t)+2 a b g^{\prime}(t)\right)^{2}+8 a b^{2} g^{\prime 2}(t)\left(-4 t g^{\prime}(t)+\right. \\
\left.2 a g^{\prime 2}(t)+4 g(t)+4 a\right)=c\left[\left(8 b^{2} g^{2}(t) g^{2}(t)-8 b^{2} a^{2} g^{\prime 2}(t)\right)\right. \\
\left(g^{\prime 2}(t)\left(4 t^{2}+4 a b g(t)+4 b g^{2}(t)\right)-\left(2 a+2 g(t)-2 t g^{\prime}(t)\right)^{2}\right)+ \\
\left.\left(8 b t g(t) g^{\prime 2}(t)-4 a b g^{\prime}(t)\left(2 a+2 g(t)-2 t g^{\prime}(t)\right)\right)^{2}\right] \\
2 g^{\prime \prime}(t)(a+g(t))\left(16 b t g(t)+8 b t^{2} g^{\prime}(t)+12 b^{2} g(t)^{2} g^{\prime}(t)+\right. \\
\left.8 a b^{2} g(t) g^{\prime}(t)\right)+8\left(b g(t) g^{\prime}(t)-b t g^{\prime 2}(t)+2 a b g^{\prime}(t)\right) \\
\left(-4 t g^{\prime}(t)+2 a g^{\prime 2}(t)+4 g(t)+4 a\right)=c\left[\left(16 b t g(t) g^{\prime 2}(t)-8 a b g^{\prime}(t)\right.\right. \\
\left.\left(2 a+2 g(t)-2 t g^{\prime}(t)\right)\right)\left(g^{\prime 2}(t)\left(4 t^{2}+4 a b g(t)\right)+\right. \\
\left.\left.4 b g(t)^{2}\right)-\left(2 a+2 g(t)-2 t g^{\prime}(t)\right)^{2}\right]
\end{gathered}
$$




$$
\begin{aligned}
& 2 g^{\prime \prime}(t)(a+g(t))\left(8 t^{2}+4 b t g(t) g^{\prime}(t)+8 a b g(t)+8 b g(t)^{2}\right)+\left(4 t g^{\prime}(t)+\right. \\
& \left.2 a g^{\prime 2}(t)+4 g(t)+4 a\right)^{2}=c\left[g^{\prime 2}(t)\left(4 t^{2}+4 a b g(t)+4 b g(t)^{2}\right)-(2 a+\right. \\
& \left.\left.2 g(t)-2 t g^{\prime}(t)\right)^{2}\right]^{2} .
\end{aligned}
$$

The only solution of the above system of equations is $g(t)=\frac{\sqrt{c}}{c}-a \neq 0$. Substituting this in relation (3.21), we obtain parametrization (3.33). Since $g(x, x)=\frac{1}{c}$, the second kind twisted surface is a pseudosphere $S_{1}^{2}\left(\frac{\sqrt{c}}{c}\right)$.

Analogously, the last theorem can be proved.

Theorem 3.10. The second kind twisted surface in $E_{1}^{3}$ with parametrization (3.21) has a non-zero constant mean curvature $H=h$, if and only if it is a part of pseudosphere $S_{1}^{2}\left(\frac{1}{h}\right)$ with parametrization

$$
\begin{aligned}
x(s, t)= & \left(\left(1-s^{2}\right)\left(t+b s\left(\frac{1}{h}-a\right)\right)+\frac{s}{h}, \frac{1}{h}-2 s\left(t+b s\left(\frac{1}{h}-a\right)\right),\right. \\
& \left.\left(1+s^{2}\right)\left(t+b s\left(\frac{1}{h}-a\right)\right)-\frac{s}{h}\right),
\end{aligned}
$$

where $a \in R, b \in R_{0}, c \in R_{0}^{+}$and $\frac{1}{h} \neq a$.

Acknowledgements. The authors are very grateful to professor Leopold Verstraelen from University of Leuven, for introducing with this topic and on his helpful comments.

\section{REFERENCES}

[1] Duggal, K.L. and Jin, D.H., Null Curves and Hypersurfaces of Semi-Riemannian Manifolds, World Scientific, Singapore, 2007.

[2] Goemans, W. and Woestyne, I. Van de, Twisted surfaces in Euclidean and Minkowski 3space, Pure and Applied Differential Geometry: 2013, J. Van der Veken, I. Van de Woestyne, L. Verstraelen and L. Vrancken (Editors), Shaker Verlag Aachen, Germany, 143-151.

[3] Goemans, W. and Woestyne, I. Van de, Constant curvature twisted surfaces in Euclidean and Minkowski 3-space, Proceedings of the conference "Riemannian Geometry and Applications to Engineering and Economics-RIGA", Bucharest, Romania, 2014, 117-130.

[4] Goemans, W. and Woestyne, I. Van de, Twisted surfaces with null rotation axis in Minkowski 3-space, Results in Math. 2015, DOI: 10.1007/ s00025-015-0462-2

[5] Gray, A., Modern Differential Geometry of Curves and Surfaces with Mathematica, CRC Press, Florida, 1998.

[6] Liu, H., Ruled surfaces with lightlike ruling in Minkowski 3 -space, J. Geom. Phys., 59 (2009), $74-78$.

[7] O'Neill, B., Semi-Riemannian Geometry with applications to relativity, Academic Press, New York, 1983.

[8] Stanilov, G., and Slavova, G., Classification of some twisted surfaces and power series of such surfaces, Comptes Rendus de l'Academie Bulgare des Sciences, 59, (2006), no. 6, 593-600.

[9] Walrave, J., Curves and surfaces in Minkowski space, Doctoral thesis, K.U. Leuven, Faculty of Science, Leuven (1995).

Department of Mathematics and Informatics, Faculty of Science, University of KRAGUJEVAC-SERBIA

E-mail address: milica_grbovic@kg.ac.rs

E-mail address: emilija@kg.ac.rs

E-mail address: anicapantic@yahoo.com 